\title{
Effect of Potential Energy Distribution on the Melting of Clusters
}

\author{
Young Joo Lee, ${ }^{1,2}$ Eok-Kyun Lee, ${ }^{1}$ and Sehun Kim ${ }^{1}$ \\ ${ }^{1}$ Department of Chemistry and School of Molecular Science (BK21), Korea Advanced Institute of Science and Technology, \\ Taejon, Korea \\ R. M. Nieminen ${ }^{2}$ \\ ${ }^{2}$ Laboratory of Physics, Helsinki University of Technology, P.O. Box 1100, FIN-02015 HUT, Finland
}

(Received 28 July 2000)

\begin{abstract}
We find that the potential energy distribution of atoms in clusters can consistently explain many important phenomena related to the phase changes of clusters, such as the nonmonotonic variation of melting temperature with size, the dependence of melting, boiling, and sublimation temperatures on the interatomic potentials, the existence of a surface-melted phase, and the absence of a premelting peak in heat capacity curves. We also find a new type of premelting mechanism in double icosahedral $\operatorname{Pd}_{19}$ clusters, where one of the two internal atoms escapes to the surface at the premelting temperature.
\end{abstract}

DOI: $10.1103 /$ PhysRevLett.86.999

PACS numbers: 36.40.Ei, 61.46.+w, 64.70.Dv

With the possibility that metal clusters could be used as building blocks for new nanomaterials [1], the understanding of the structures and the thermal stabilities of metal clusters has become important. Recent experimental measurements of the heat capacities for unsupported metal clusters by Schmidt and co-workers [2,3] have given a strong motivation for the atomistic understanding of the melting of metal clusters. It has been observed both in experiments [4] and in theoretical simulations [5] that clusters melt at temperatures lower than those of bulk melting due to the high proportion of surface atoms with lower binding energies. As the cluster size decreases, the melting temperature monotonically decreases $[4,5]$. However, when the cluster size is small enough ( $\leq 200$ atoms), the melting temperature does not vary monotonically with the size of cluster $[3,6,7]$.

Multimodal heat capacity curves observed in small clusters indicate that thermal energy can be dissipated by a "premelting" mechanism before overall melting [7]. Premelting has been attributed to surface melting [8], partial melting [9], orientational disordering in molecular cluster [10], and isomerization [7,11]. It has been observed that clusters can dissipate thermal energy even by boiling [12] and sublimation [13]. In some clusters, the premelting peak in the heat capacity curve is not observed $[9,14-16]$.

Previously published simulations of cluster melting have used either Monte Carlo (MC) methods at constant temperature (canonical ensemble) or molecular dynamics (MD) at constant energy (microcanonical ensemble). There has been some controversy concerning the dependence of the simulated thermodynamic properties on the ensemble until the work by Calvo and Labastie [17]. These authors [17] showed that if the sampling of the phase space is accurate enough (a large enough number of MC configurations and MD trajectories), the thermodynamic results are identical in both ensembles because the thermodynamics is determined by the configurational density of states $[7,8,17,18]$.
In this Letter, we show that the potential energy distribution of atoms in the clusters can consistently explain many of the important phenomena which occur during phase changes of small clusters, such as the nonmonotonic variation of melting temperature with the size of clusters $[3,6,7]$, the dependence of melting, boiling, and sublimation temperatures on the interatomic potentials $[12,13,19]$, the existence of a surface-melted phase $[6,8,11,15,16]$, and the absence of a premelting peak in heat capacity curves [9,14-16].

We have studied the thermodynamic behavior of clusters of metals and nonmetals for the sizes of $12 \leq n \leq 34$. For the interatomic interactions, we have employed the tight-binding potentials based on the second moment approximation [7,16] for metal clusters $\left(M_{n}, M=\mathrm{Ni}, \mathrm{Cu}\right.$, $\mathrm{Pd}, \mathrm{Ag}, \mathrm{Pt}, \mathrm{Au}, \mathrm{Al}$, and $\mathrm{Pb}$ ) and pair potentials for nonmetal clusters. The tight-binding model for the interatomic interactions has been tested against both experiment and $a b$ initio calculations. The agreement with experimental structures for $\mathrm{Ni}$ and $\mathrm{Cu}$ clusters $[20,21]$ and with $a b$ initio results for $\mathrm{Au}[22]$ is good.

To search for the lowest-energy structures, simulated annealing and quenching by MC methods are extensively applied to thousands of random configurations and to large numbers of probable structures based on icosahedral, decahedral, and close packing. In the simulations of melting, we monitor the phase changes of clusters using atom-resolved quantities [16] such as the average potential energy $\left\langle E_{i}\right\rangle$ of an atom $i$ and the root-mean-square fluctuation of the interatomic distances,

$$
\left\langle\delta_{i}\right\rangle=\frac{1}{N-1} \sum_{j \neq i}^{N} \frac{\left(\left\langle r_{i j}^{2}\right\rangle-\left\langle r_{i j}\right\rangle^{2}\right)^{1 / 2}}{\left\langle r_{i j}\right\rangle} .
$$

The atom-resolved $\left\langle\delta_{i}\right\rangle$ measures the extent of melting of an individual atom just as the Lindemann index $\langle\delta\rangle$ measures the extent of melting of the entire cluster. 
Simulations of melting are performed by the standard isothermal Metropolis MC (MMC) method, even though the $J$-walking $\mathrm{MC}$ and the $q$-jumping MC methods [23] are more efficient in overcoming the barrier between the local minima. In both the $J$-walking and the $q$-jumping MC methods, the atom-resolved quantities are not generally meaningful because there is no continuous evolution of the connectivity of atoms over the entire cycles of MC simulation [23]. We have made stringent tests for the convergence of thermodynamics by increasing the number of MC steps (MCS) up to $10^{9}$, where one MCS means sampling all atoms once on the average. The heat capacity curves calculated by the MMC and $J$-walking MC methods are almost identical as long as the number of samples is larger than $5 \times 10^{6}$ MCS.

The most stable structure is an icosahedron with one vacancy for all $\mathrm{M}_{12}$ clusters (except for $\mathrm{Pt}_{12}$ and $\mathrm{Au}_{12}$ which have nonspherical glasslike structures without internal atom), an icosahedron for $\mathrm{M}_{13}$, a capped icosahedron for $\mathrm{Ni}_{14}, \mathrm{Cu}_{14}, \mathrm{Pd}_{14}, \mathrm{Ag}_{14}$, and $\mathrm{Pb}_{14}$, a distorted structure with one atom inserted on the surface for $\mathrm{Al}_{14}$, and a Frank-Kasper structure with one vacancy on the surface for $\mathrm{Pt}_{14}$ and $\mathrm{Au}_{14}$. For $\mathrm{M}_{19}$ clusters, the most stable structure is a double icosahedron with two atoms inside the cluster for $\mathrm{Ni}_{19}, \mathrm{Cu}_{19}, \mathrm{Pd}_{19}, \mathrm{Ag}_{19}$, and $\mathrm{Al}_{19}$, but a glasslike structure with only one internal atom with the coordination number of 16 for $\mathrm{Pt}_{19}, \mathrm{Au}_{19}$, and $\mathrm{Pb}_{19}$. For $\mathrm{M}_{23}$ clusters, the most stable structure is a triple icosahedron with three atoms inside the cluster for $\mathrm{Ni}_{23}$ and $\mathrm{Cu}_{23}$, and a largely distorted glasslike structure with two atoms inside the cluster for $\mathrm{Pd}_{23}, \mathrm{Ag}_{23}, \mathrm{Pt}_{23}, \mathrm{Au}_{23}, \mathrm{Al}_{23}$, and $\mathrm{Pb}_{23}$.

Some representative heat capacity curves of metal clusters are shown in Figs. 1(a) and 1(b). The heat capacity curves show the typical rounded first-order-like phase change due to the finite size effect for all metal clusters. All $\mathrm{M}_{14}$ clusters, with the exception of $\mathrm{Ni}_{14}$, show an additional peak at a temperature lower than that for the maximum peak. The $\mathrm{Pd}_{19}$ cluster shows a distinct abrupt change at $400 \mathrm{~K}$ and rounded-off broad peak at a higher temperature. As the cluster size increases from $n=12$ to $n=23$, the overall melting temperature $\left(T_{m}\right)$ determined by the heat capacity maximum varies nonmonotonically as shown in Fig. 1(c). When the cluster melting temperatures are normalized by the experimental bulk melting temperatures, a strong dependence of melting temperatures on the element is observed as shown in Fig. 1(d). The dependence of $T_{m}$ on the potentials was reported for $\mathrm{Ni}_{13}$ and $\mathrm{Au}_{13}$ by Garzon and Jellinek [19].

We analyze the cluster melting phenomenon using the distribution of potential energies of individual atoms. We classify the atom in the cluster to three types depending on their coordination number $(\mathrm{CN})$ which is the number of nearest neighbors within a cutoff radius equal to $1.2 r_{0}\left(r_{0}\right.$ is the nearest neighbor distance in the perfect crystal): an internal atom with $\mathrm{CN} \geq 11$, a capped atom with $\mathrm{CN}=$ $\sim 3-4$, and a surface atom with $\mathrm{CN}=\sim 5-10$. The word

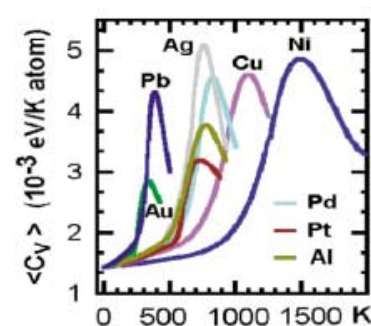

(a)

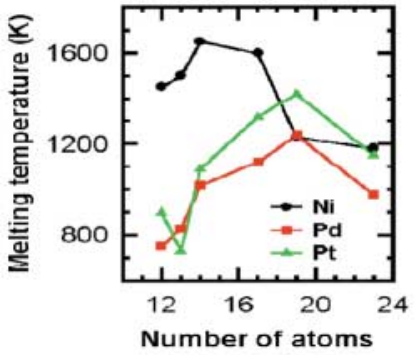

(c)

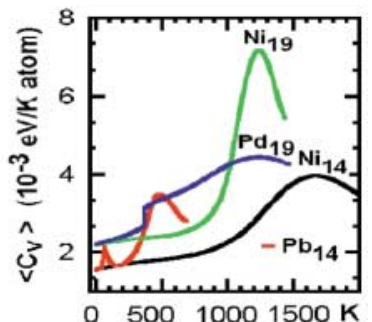

(b)

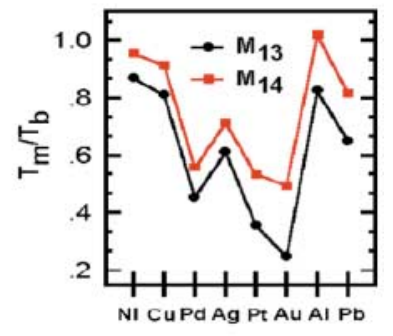

(d)
FIG. 1 (color). Calculated heat capacities of (a) $\mathrm{M}_{13}$, (b) $\mathrm{Ni}_{14}$, $\mathrm{Pb}_{14}, \mathrm{Ni}_{19}$, and $\mathrm{Pd}_{19}$. (c) Overall melting temperatures versus cluster size. (d) Overall melting temperatures normalized by experimental bulk melting temperatures.

of surrounding atoms is used to represent both the surface and capped atoms.

Figure 2 shows the evolution of the mean potential energy for different types of atoms as a function of the equilibrium temperature. The distributions of potential energies $\left\langle E_{i}\right\rangle$ for the initial structures are very different for different clusters even though the structures are the same at zero temperature. As the temperature increases, the potential energies of individual atoms in the cluster redistribute in different ways. The melting starts at the temperature where the colors cannot be discerned due to the thermal distortion and the frequent site exchange between atoms. The premelting is observed at $200 \mathrm{~K}$ for $\mathrm{Ni}_{14}, 60 \mathrm{~K}$ for $\mathrm{Pb}_{14}, 750 \mathrm{~K}$ for $\mathrm{Ni}_{19}$, and $400 \mathrm{~K}$ for $\mathrm{Pb}_{19}$, respectively. It should be noted that $\mathrm{Ni}_{14}$ and $\mathrm{Ni}_{19}$ clusters in which the internal atoms are more stable than the surrounding atoms do not show the expected premelting peak on their heat capacity curves, while the other clusters in which the internal atoms are less stable than the surrounding atoms show the expected premelting peak [see Fig. 1(b)].

We define the relative stability of the internal atoms to the surrounding atoms in the cluster by the parameter $S=\bar{E}_{\text {int }} / \bar{E}_{\text {sur }}$, where $\bar{E}_{\text {int }}$ and $\bar{E}_{\text {sur }}$ are the mean potential energies of the internal atoms and the surrounding atoms, respectively. If $S$ is larger than 1 , the surface atom is less stable than the internal atom, and if $S$ is less than 1, the surface atom is more stable than the internal atom.

As for premelting, we observe two different mechanisms depending on the value of the parameter $S$. If $S \geq 1.15$ (as for $\mathrm{Ni}_{14}$ and $\mathrm{Ni}_{19}$ ), the premelting takes place by permutational isomerization which does not give rise to a 

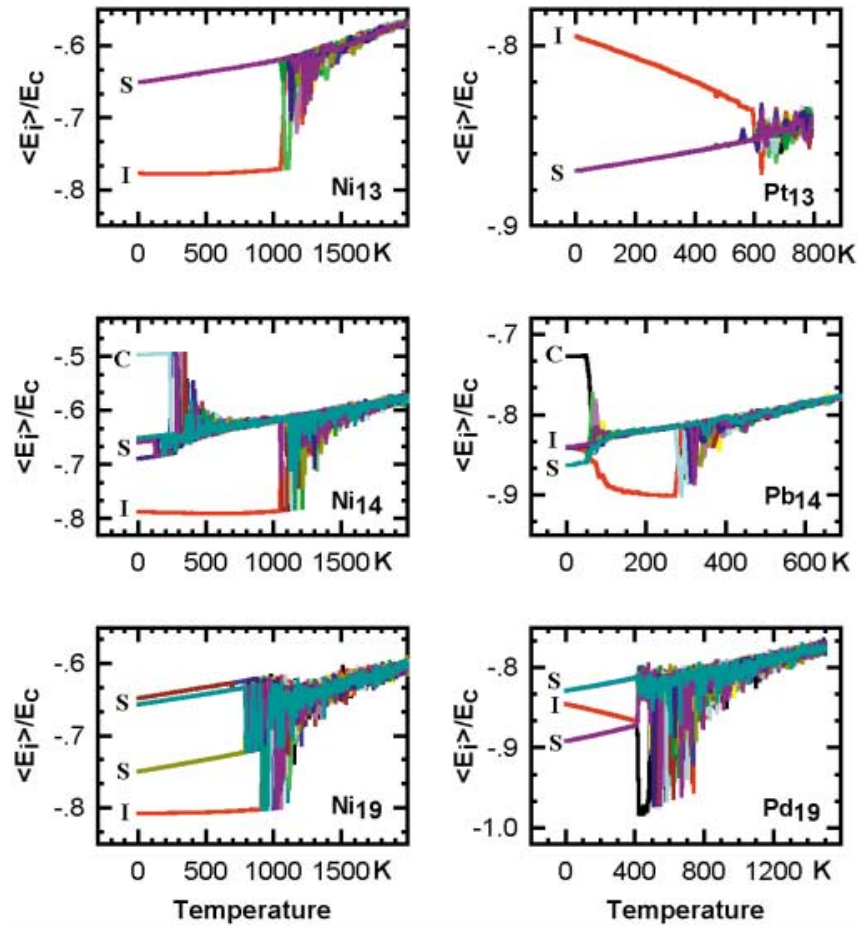

FIG. 2 (color). Potential energy change of each atom as a function of temperature, where the $E_{c}$ is cohesive energy of bulk. The labels I, S, and C represent the internal atom, the surface atom, and the capped atom.

premelting peak, because the energies of permutational isomers are the same [16] [see Fig. 1(b)]. If $S<1.15$ (as for $\mathrm{Pb}_{14}$ and $\mathrm{Pb}_{19}$ ), premelting proceeds by geometrical isomerization and gives rise to a premelting peak due to energy fluctuations [see Fig. 1(b)]. The high potential energy of the internal atom in the capped icosahedral $\mathrm{Pb}_{14}$ at zero temperature is lowered by inserting the capped atom into the surface layer at the premelting temperature. The high potential energies of the internal atoms in the double icosahedral $\mathrm{Pd}_{19}$ are lowered by a mechanism where one of the two internal atoms is ejected to the surface resulting in a highly distorted glasslike structure with only one internal atom (see Fig. 2). The premelting in $\mathrm{Pd}_{19}$ is initiated by the transition of the double icosahedron with two internal atoms to the glasslike structures with one internal atom. The value of $S \geq 1.15$ can also be used to explain the absence of the premelting peak in the heat capacity curves of the clusters modeled by other $n$-body potentials [9] as well as pair potentials $[8,15]$.

The atom-resolved Lindemann index $\left\langle\delta_{i}\right\rangle$ in Figs. 3(a)-3(c) clearly shows the different melting behaviors depending on the value of $S$. If the surrounding atoms are less stable than the internal atom $(S \geq 1)$, the clusters melt starting with the surrounding atoms, resulting in the surface-melted phase, as shown in Figs. 3(a) and 3(c) for $\mathrm{Ni}_{13}$ and $\mathrm{Pb}_{14}$, irrespective of the size and geometry of clusters. However, if the surrounding atoms are more stable than the internal atom $(S \leq 1)$, the clusters
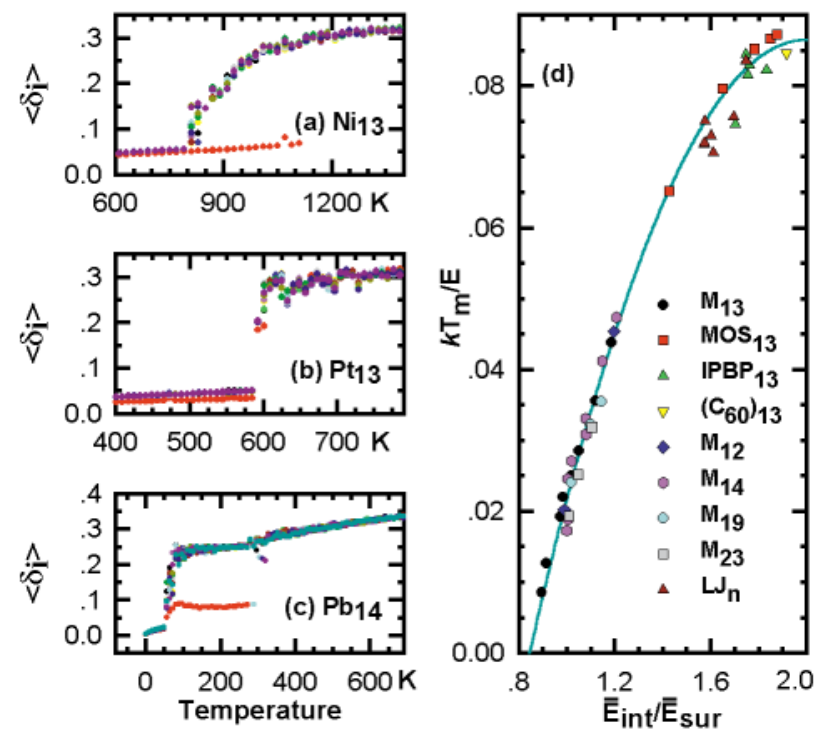

FIG. 3 (color). (a),(b),(c) Root-mean-square fluctuation of interatomic distance $\left\langle\delta_{i}\right\rangle$ versus temperature. The red color represents the internal atoms below the overall melting temperature. (d) Overall melting temperature normalized by the cluster binding energy $E$ versus $S$. The details of the size and species of the clusters are described in Ref. [24].

start to melt homogeneously, as shown in Fig. 3(b) for $\mathrm{Pt}_{13}$. These clusters do not show the surface-melted phase.

Surface melting can be explained by the weaker binding energy of surface atom compared to the internal atom. The weakly bound surface atoms can easily move and form hole-floater structures before the stable internal atoms are involved in melting [16]. The criterion of $S \geq 1$ can also be used to explain the surface melting observed in other clusters modeled by Lennard-Jones potentials $[6,8]$, the inverse-power-based potentials [15], and Gupta potentials [11], for example. The values of $S$ for these clusters are in the range of 1.0-1.7.

As the value of $S$ increases, the binding energy of surface atoms becomes weaker. In the clusters with a very high value of $S$, boiling and sublimation can occur near the melting temperature and below the melting temperature, respectively, due to the very weakly bound surface atoms. In fact, boiling [12] and sublimation [13] have been observed in the clusters modeled by Morse potentials [12] and in fullerene clusters $\left(\mathrm{C}_{60}\right)_{13}$ modeled by the Girifalco potential [13], respectively. For these clusters the calculated values of $S$ are 1.88 and 1.92, respectively. We suggest the criterion of $S \geq 1.9$ for the observation of sublimation before melting.

The effect of the relative potential energy, $S$, on the overall melting temperature of clusters is investigated for various types of clusters (see the footnote [24] for the size and species of the clusters). Figure 3(d) shows that the overall melting temperature normalized by the cluster binding energy $E$ increases as the value of $S$ increases, irrespective of the details of potentials and the size of clusters. 
The value of $S$ is averaged for the isomers with potential energies less than $0.001 \mathrm{eV} /$ atom above the most stable structure. Since cluster melting completes the process by involving the internal atom in the melting, the overall melting temperature increases as the binding energy of the internal atom increases. When the experimental melting temperatures [3] by Schmidt and co-workers are normalized by the binding energies calculated for the stable structures, the normalized experimental melting temperatures are $0.023-0.027$, which are in the range of our calculated values of 0.01-0.09 for transition metal, simple metal, and nonmetal clusters. As the value of $S$ decreases, the normalized melting temperature eventually approaches zero at the value of $S=0.85$, which means that the liquidlike disordered structures are more stable than the solidlike ordered structures at zero temperature. The value $S=0.85$ is surprisingly close to the value $S=0.849$ for the icosahedral $\mathrm{Pt}_{13}$ obtained by Sachdev et al. [25]. For the $\mathrm{Pt}_{13}$ clusters modeled by embedded atom method, the liquidlike nonspherical structures with no internal atom are more stable than the solidlike icosahedral cluster. We suggest the value of $S \leq 0.85$ as the criterion for the zero-temperature melting of icosahedral 13-atom clusters.

The observed universal curve of melting temperatures versus $S$ [Fig. 3(d)] implies that the nonmonotonic variation of melting temperature with the cluster size [Fig. 1(c)] is due to the nonmonotonic variation of the value of $S$ with the size, and the dependence of melting temperatures on the potentials [Fig. 1(d)] is due to the different distributions of the potential energies of atoms in the clusters.

In conclusion, we explain consistently many important phenomena related to the phase changes of clusters, such as the nonmonotonic variation of melting temperature with size, the dependence of melting, boiling, and sublimation temperatures on the potentials, the existence of a surface-melted phase, and the absence of a premelting peak in heat capacity curves in terms of the potential energy distribution.

[1] S. Chen, R. S. Ingram, M. J. Hostetler, J. J. Pietron, R. W. Murray, T. G. Schaaff, J. T. Khoury, M. M. Alvarez, and R. L. Whetten, Science 1998, 2098 (1998).

[2] M. Schmidt, R. Kusche, W. Kronmüller, B. von Issendorff, and H. Haberland, Phys. Rev. Lett. 79, 99 (1997).

[3] M. Schmidt, R. Kusche, B. von Issendorff, and H. Haberland, Nature (London) 393, 238 (1998).

[4] S. L. Lai, J. Y. Guo, V. Petrova, G. Ramanath, and L. H. Ailen, Phys. Rev. Lett. 77, 99 (1996).
[5] F. Ercolessi, W. Andreoni, and E. Tosatti, Phys. Rev. Lett. 66, 911 (1991).

[6] T. L. Beck, J. Jellinek, and R. S. Berry, J. Chem. Phys. 87, 545 (1987).

[7] F. Calvo and F. Spiegelmann, Phys. Rev. Lett. 82, 2270 (1999); J. Chem. Phys. 112, 2888 (2000).

[8] F. Calvo and P. Labastie, Chem. Phys. Lett. 258, 233 (1996).

[9] C. Rey, L. J. Gallego, J. C. Rodeja, J. A. Alonso, and M. P. Iñiguez, Phys. Rev. B 48, 8253 (1993); J. G. Rodeja, C. Rey, J. L. Gallego, and J. A. Alonso, Phys. Rev. B 49, 8495 (1994); (private communication).

[10] J.-B. Maillet, A. Boutin, and A. H. Fuchs, Phys. Rev. Lett. 76, 4336 (1996).

[11] E. B. Krissinel and J. Jellinek, Int. J. Quantum Chem. 62, 185 (1997).

[12] M. Moseler and J. Nordiek, Phys. Rev. B 60, 11734 (1999); (private communication).

[13] C. Rey, L. J. Gallego, and J. A. Alonso, Phys. Rev. B 49, 8491 (1994).

[14] J. Jellinek and I. L. Garzón, Z. Phys. D 20, 239 (1991).

[15] C. Rey, J. G. Rodeja, L. J. Gallego, and M. J. Grimson, Phys. Rev. E 57, 4420 (1998); (private communication).

[16] Y. J. Lee, J. Y. Maeng, E.-K. Lee, B. Kim, S. Kim, and K.-K. Han, J. Comput. Chem. 21, 380 (2000).

[17] F. Calvo and P. Labastie, Chem. Phys. Lett. 247, 395 (1995).

[18] P. Labastie and R. L. Whetten, Phys. Rev. Lett. 65, 1567 (1990).

[19] I. L. Garzon and J. Jellinek, Z. Phys. D 26, 316 (1993).

[20] E. K. Parks, L. Zhu, J. Ho, and S. J. Riley, J. Chem. Phys. 100, 7206 (1994); 102, 7377 (1995).

[21] D. Reinhard, B. D. Hall, P. Berthoud, S. Valkealahti, and R. Monot, Phys. Rev. Lett. 79, 1459 (1997).

[22] I. L. Garzón, K. Michaelian, M. R. Beltrán, A. P. Amarillas, P. Ordejón, E. Artacho, D. S. Portal, and J. M. Soler, Phys. Rev. Lett. 81, 1600 (1998).

[23] D. D. Frantz, J. Chem. Phys. 102, 3747 (1995); I. Andricioaei and E. Straub, J. Chem. Phys. 107, 9117 (1997).

[24] The melting temperatures are plotted for the clusters as follows: $\mathrm{Ni}$ and $\mathrm{Pd}$ for $\mathrm{M}_{12}$. Ni, $\mathrm{Cu}, \mathrm{Pd}, \mathrm{Ag}, \mathrm{Pt}, \mathrm{Au}, \mathrm{Al}, \mathrm{Pb}$ for $\mathrm{M}_{13}$ and $\mathrm{M}_{14}$. Ni, Pd, Pt for $\mathrm{M}_{19}$ and $\mathrm{M}_{23}$. Lennard-Jones clusters $\left(\mathrm{LJ}_{n}\right)$ for the sizes of 13, 19, 23, 26, 29, 32, and 34. Thirteen-atom Morse clusters $\left(\operatorname{MOS}_{13}\right)$ for the interatomic potentials with $\rho_{o}=3, \rho_{o}=4, \rho_{o}=5, \rho_{o}=6$, $\rho_{o}=14$. Thirteen-atom clusters modeled by the inverse power based potentials $\left(\mathrm{IPBP}_{13}\right)$ of $m-n=7-1,8-2,9-3$, 10-4, 12-6, and 20-14. Fullerene cluster, $\left(\mathrm{C}_{60}\right)_{13}$, modeled by the Girifalco potential. For the nonmetal clusters of $\mathrm{LJ}_{n}$, $\mathrm{MOS}_{13}, \mathrm{IPBP}_{13}$, and $\left(\mathrm{C}_{60}\right)_{13}$, the melting temperatures are from Refs. [8,18], Ref. [12], Ref. [15], and Ref. [13], respectively.

[25] A. Sachdev, R. I. Masel, and J. B. Adams, J. Catal. 136, 320 (1992). 